\section{Adjuvante Chemo im Stadium II und bei Älteren ohne Platin}

\section{Seit der MOSAIC-Studie (2004) ist im Stadium III des Kolonkarzinoms die adjuvante Therapie mit 5-Fluorouracil(5-FU)/Leucovorin plus Oxaliplatin Standard. Im Stadium II wird die routinemäßige adjuvante Chemotherapie nicht empfohlen; die Studienlage ist hier widersprüchlich.}

$\mathrm{n}$ der MOSAIC-Studie hatten bis 75-jährige Patienten im Stadium II oder III randomisiert Folinsäure, gefolgt von 5-FU als Bolus und danach als Infusion über 22 Stunden an zwei Tagen (FL) ohne oder mit Oxaliplatin (FOLFOX4) erhalten. In einer Post-hoc-Analyse wurden nun Therapieergebnisse und Verlauf bei Patienten mit 70 oder mehr Jahren (n = 315) sowie mit Tumoren im Stadium II $(\mathrm{n}=899)$ untersucht. Von den Teilnehmern mit Stadium-II-Tumoren wurden 569 als Hochrisiko-Patienten klassifiziert (T4, Tumorperforation, Ileus, schlecht differenzierter Tumor und/oder eine $\mathrm{zu}$ geringe Zahl untersuchter Lymphknoten $[<10]$ ). Bezüglich des krankheitsfreien Überlebens (primärer Endpunkt) betrug die Hazard Ratio [HR] bei diesen Patienten im FOLFOXArm gegenüber dem Vergleichsarm 0,84 $(\mathrm{p}=0,258)$, bezüglich der Zeit bis zum Auftreten eines Rezidivs 0,70 ( $\mathrm{p}=0,045)$. Das Gesamtüberleben war in beiden Kollektiven gleich: Die 6-Jahres-Überlebensraten beliefen sich auf 85 vs. 83,3\%.

Die mediane Überlebensdauer nach Erreichen des Endpunkts krankheitsfreies Überleben betrug unter der Oxaliplatin-haltigen Therapie 13,4 Monate gegenüber 28,6 in der Vergleichsgruppe.

Patienten mit Low-Risk-Karzinom zogen keinen Zusatznutzen aus der Oxaliplatin-haltigen Therapie. Bei Hochrisi-
ko-Konstellation wurde durch Oxaliplatin zwar die Zeit bis zum Rezidiv verlängert (HR 0,62; $p=0,02)$, aber weder die krankheitsfreie Überlebensdauer noch die Gesamtüberlebenszeit.

Ähnliches ergab die Analyse für die kleine Zahl der Patienten im Alter von 70-75 Jahren. Diese lebten nach Erreichen des Endpunkts krankheitsfreies Überleben unter dem FOLFOX-Regime im Median noch 3,6 Monate - im Vergleich zu 13,7 Monaten im Parallel-Arm.

Fazit: Weder im Stadium II noch bei über 70-Jährigen fand sich in der Subgruppen-Analyse unter Oxaliplatin-Zusatz ein signifikanter Vorteil, was das krankheitsfreie wie das Gesamtüberleben angeht.

Waltraud Paukstadt

Tournigand $C$ et al. Adjuvant therapy with fluorouracil and oxaliplatin in stage II and elderly patients (between ages 70 and 75 years) with colon cancer: Subgroup analyses of the Multicenter International Study of Oxaliplatin, Fluorouracil, and Leucovorin in the Adjuvant Treatment of Colon Cancer Trial. J Clin Oncol. 2012; 30(27):3353-60.

\section{Auch über 75-Jährigen nützt die Chemotherapie}

\section{Etwa $40 \%$ der Patienten mit einem kolorektalen Karzinom sind mindestens 75 Jahre alt. Dennoch ist diese Altersgruppe in klinischen Studien unter- repräsentiert. In einer Analyse von Registerdaten wurde nun untersucht, ob eine Chemotherapie das Leben älterer Patienten mit Kolonkarzinom verlängern kann.}

E ine adjuvante Chemotherapie kann die Heilungschancen von Patienten mit Kolonkarzinom im Stadium III, bei denen eine Operation mit kurativer Absicht vorgenommen wurde, verbessern. Die Therapie besteht in der Regel aus Fluorouracil, Capecitabin oder der Kombination von Fluorouracil oder Capecitabin mit Oxaliplatin.

Um den Nutzen einer adjuvanten Chemotherapie mit und ohne Oxaliplatin bei älteren Patienten mit Kolonkarzinom im Stadium III beurteilen zu können, wurden Daten aus vier großen USamerikanischen Krebsregistern ausgewertet. In die Analyse wurden $5.489 \mathrm{~Pa}$ tienten aufgenommen, die zum Zeitpunkt der Diagnose mindestens 75 Jahre alt waren, in den vorhergehenden 90
Tagen operiert worden waren und mindestens 30 Tage nach der Operation noch gelebt hatten.

Patienten, die innerhalb von $120 \mathrm{Ta}$ gen nach der Resektion starben, waren deutlich älter als Patienten, die diesen Zeitraum überlebten. Nur wenige der verstorbenen Patienten hatten eine Chemotherapie erhalten.

Der Anteil der Patienten, die eine Chemotherapie erhalten hatten, variierte in den vier Kohorten von $42-75 \%$, wobei nur ein Teil der Patienten Oxaliplatin bekam. Erwartungsgemäß kam eine Chemotherapie mit zunehmendem Alter immer seltener zum Einsatz. Während von den 75-79-Jährigen noch $63 \%$ eine Chemotherapie bekamen, waren dies bei den 80-84-Jährigen nur noch
$43 \%$ und in der Gruppe ab 85 Jahren nur noch $14 \%$.

Patienten mit Chemotherapie lebten deutlich länger als Patienten ohne Chemotherapie. Die Mortalität sank durch die Chemotherapie in den vier Kohorten um 24-58\% im Vergleich zu Patienten ohne Chemotherapie. Dabei war der Überlebensvorteil der älteren Patienten ähnlich hoch wie in klinischen Studien mit jüngeren Patienten. Bei Anwendung von Oxaliplatin war ein Trend zu einer niedrigeren Mortalität im Vergleich zur Chemotherapie ohne Oxaliplatin zu beobachten. Allerdings gab es hier keine einheitlichen Ergebnisse hinsichtlich der statistischen Signifikanz.

Fazit: Diese Untersuchung zeigt, dass eine adjuvante Chemotherapie das Leben älterer Patienten mit Kolonkarzinom im Stadium III verlängern kann. Oxaliplatin bietet allerdings nur geringen zusätzlichen Nutzen. Judith Neumaier

Sanoff HK et al. Effect of adjuvant chemotherapy on survival of patients with stage III colon cancer diagnosed after age 75 Years. J Clin Oncol. 2012;30(21):2624-34. 